\title{
INSAR FORENSICS: TRACING INSAR SCATTERERS IN HIGH RESOLUTION OPTICAL IMAGE
}

\author{
Yuanyuan Wang ${ }^{(1)}$, Xiao Xiang Zhu ${ }^{(1,2)}$ \\ ${ }^{(1)}$ Helmholtz Young Investigators Group "SiPEO", Technische Universität München, Arcisstraße 21, 80333 Munich, \\ Germany.Email: wang@bv.tum.de \\ (2) Remote Sensing Technology Institute (IMF), German Aerospace Center (DLR), Oberpfaffenhofen, 82234 Weßling, \\ Germany.Email: xiao.zhu@dlr.de
}

\begin{abstract}
This paper presents a step towards a better interpretation of the scattering mechanism of different objects and their deformation histories in SAR interferometry (InSAR). The proposed technique traces individual SAR scatterer in high resolution optical images where their geometries, materials, and other properties can be better analyzed and classified. And hence scatterers of a same object can be analyzed in group, which brings us to a new level of InSAR deformation monitoring.
\end{abstract}

\section{INTRODUCTION}

Large area deformation monitoring is so far only achievable through SAR interferometry (InSAR) techniques such as persistent scatterer interferometry (PSI) and SAR tomography (TomoSAR). Through modelling the interferometric phase of the scatterers, we are able to reconstruct their 3-D positions and the deformation histories. However, the current SAR theory makes a quite restrictive assumption - linearity - in the imaging model, for the convenience of mathematical derivation. That is to say the imaged area is considered as an ensemble of individual point scatterers whose scattered fields and, hence, their responses in the SAR image superimpose linearly [1]. In the reality, the true position and the exact scattering mechanism of the scatterer still require further study.

This work presents a step towards a better understanding of the scattering mechanism of different objects. We back trace individual SAR scatterer in high resolution optical images where we can analyze the semantics and other properties of the imaged object.

This work is towards a future generation of InSAR techniques that are contextually aware of the semantics in a SAR image, which enables the object-level deformation reconstruction and analysis from SAR images, instead of the current pixel-based reconstruction without the understanding of the manmade world that is imaged. The proposed approach brings the first such analysis via a semantic classification in the InSAR point cloud.

The general framework of the proposed approach is shown in Figure 1. The semantic classification of the InSAR point cloud is achieved by co-registering the InSAR point cloud and an optical image to a common reference 3-D model, so that the semantic classification in the optical image can be transfer to the InSAR point cloud. The general procedures are as follows.

a. Retrieve the 3-D positions of the scatterers from SAR image stacks. Since urban area is of our main interest, tomographic SAR inversion should be employed in order to resolve a substantial amount of layovered scatterers.

b. Absolute georeference the 3-D InSAR point cloud, due to the relative position of the InSAR point cloud w.r.t. a reference point. This step is achieved by co-registering the InSAR point cloud with a reference 3-D model.

c. Texturing the reference 3-D model with high resolution optical images, so that each SAR scatterer can be traced in the optical image.

d. Classify the optical image pixels based on its semantic meaning, e.g. geometry, material, and so on.

e. Perform further analysis on object-level in the InSAR point cloud based on their semantic class.

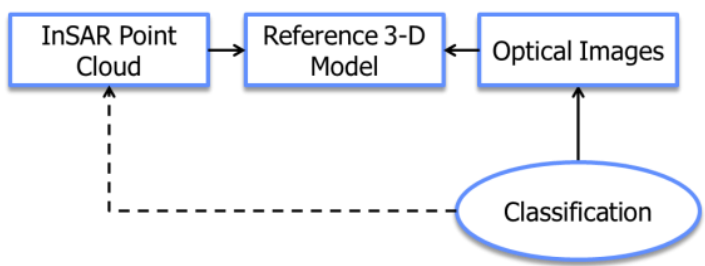

Figure 1. Flowchart of the proposed method. The semantic classification of the InSAR point cloud is achieved by coregistering the InSAR point cloud and the optical image to a reference model.

\section{TOMOGRAPHIC SAR INVERSION}

The SAR tomography (TomoSAR) processing aims at separating multiple scatterers possibly layovered in the same pixel, and retrieving their third coordinate elevation in the SAR native coordinate system. Displacement of the scatterers can also be modeled and estimated, using stack of images acquired at different times. This is commonly known as differential SAR tomography (D-TomoSAR) [2]-[4]. 
We make use of the D-TomoSAR software TomoGENESIS [5], [6] developed in DLR to process TerraSAR-X image stacks. For an input data stack, Tomo-GENESIS retrieves the following information:

- the number of scatterers inside each pixel,

- the scattering amplitude and phase of each scatterer,

- $\quad$ and their 3D positions and motion parameters, e.g. linear deformation rate and amplitude of seasonal motion.

The scatterers' 3D positions in SAR coordinates can be converted into a local Cartesian coordinate system, such as Universal Transverse Mercator (UTM), so that the results from multiple data stacks with different viewing angles can be combined. For our test area Berlin, two image stacks - one ascending orbit, the other descending orbit - are processed. These two point clouds are fused to a single one, using a feature-based matching algorithm which estimates and matches common building edges in the two point clouds [7]. The following figure is the fused point cloud which provides a complete monitoring over the whole city of Berlin.

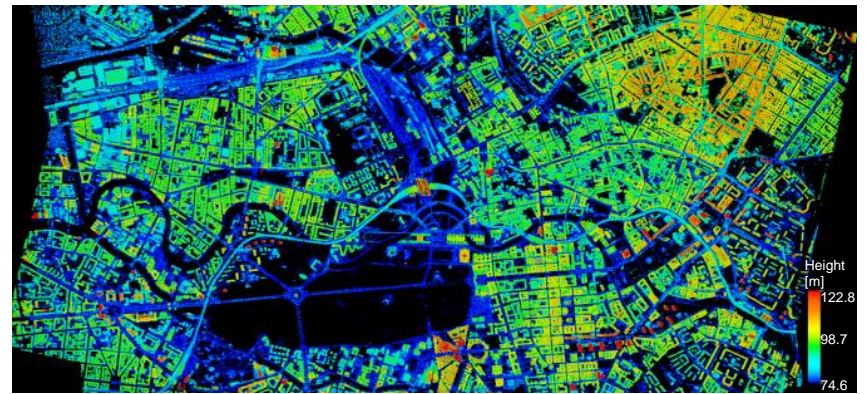

Figure 2. The fused TomoSAR point cloud of Berlin, which combines the result from an ascending stack and a descending stack. The height is color-coded.

\section{COREGISTRATION OF TOMOSAR POINT CLOUDS AND THE REFERENCE MODEL}

\subsection{CO-REGISTRATION WORKFLOW}

Our reference model is a $3 \mathrm{D}$ point cloud from an airborne LiDAR sensor [8], which is represented, same as the TomoSAR point cloud, in the UTM coordinate system. And hence, the co-registration problem is the estimation of translation between two rigid point clouds, subject to a certain tolerance on rotation and scaling. However, our LiDAR point cloud is nadir-looking, in contrast to the side-looking geometry of SAR. In another word, façade point barely appears in LiDAR point cloud while it is prominent in TomoSAR point cloud. This difference is exemplified in Figure 3, where the left and the right subfigures correspond to the TomoSAR and LiDAR point clouds of the same area. These unique modalities have driven our algorithm developed in the following way:
1 Edge extraction

a. The LiDAR point cloud is rasterized into a 2D height image.

b. The point density of TomoSAR point cloud is estimated on the rasterized 2D grid.

c. The edges in the LiDAR height image and the TomoSAR point density image are detected.

2 Initial alignment

a. Horizontally by cross-correlating the two edge images.

b. Vertically by cross-correlating the height histogram of the two point clouds.

3 Refined solution

a. The façade points in both point clouds are removed.

b. The final solution is obtained using iterative closest point (ICP) applied on the two reduced point clouds.

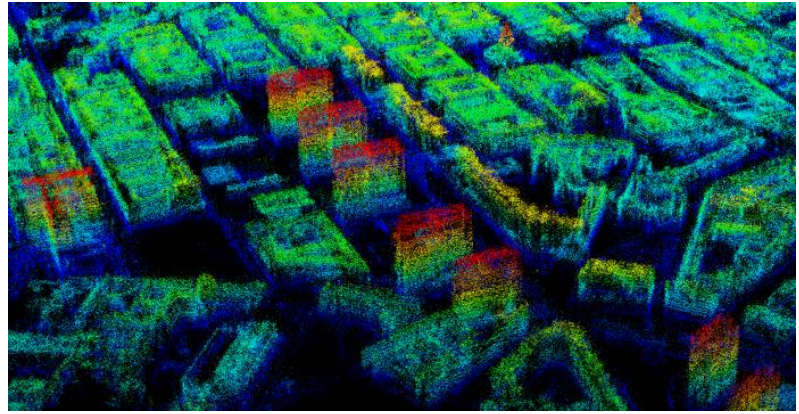

(a)

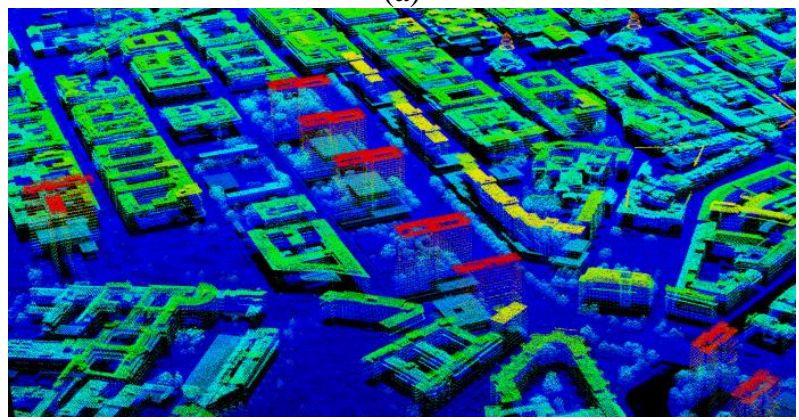

(b)

Figure 3. (a) TomoSAR point cloud of high-rise buildings, and (b) the LiDAR point cloud of the same area. Building façades are almost invisible in the LiDAR point cloud, while it is prominent in the TomoSAR point cloud.

\subsection{2-D EDGE EXTRACTION}

In order to obtain the height image and the point density image of LiDAR and TomoSAR point clouds respectively, the two point clouds are tiled according to a $2 \mathrm{D}$ grid. Here we use $2 \times 2 \mathrm{~m}$ for our dataset. For the LiDAR point cloud, the mean height in each grid cell is computed, while for the TomoSAR point cloud, the number of points inside the grid cell is counted. The edges can be extracted from these two images using any edge detector, such as Sobel filter [9]. The thresholds in the edge detector are decided adaptively, so that the 
numbers of edge pixels in the two edge images are on the same scale. The following figure is a close up view of the two edge images near downtown Berlin.

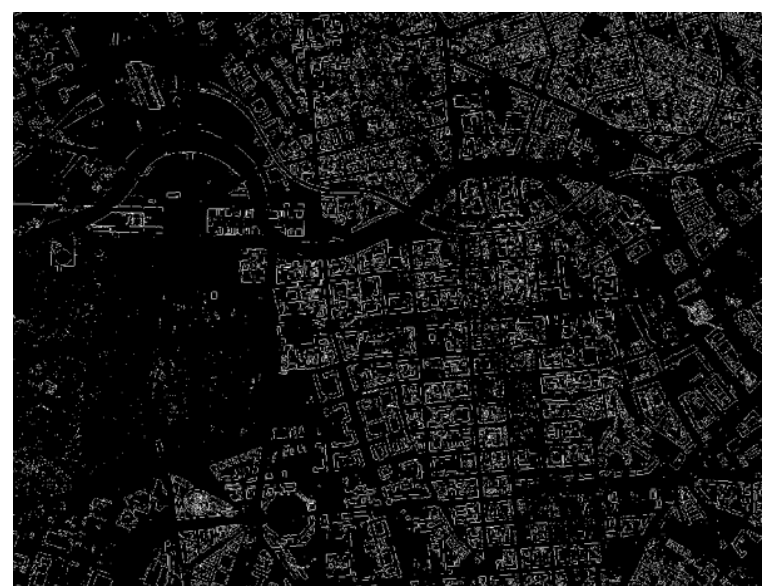

(a)

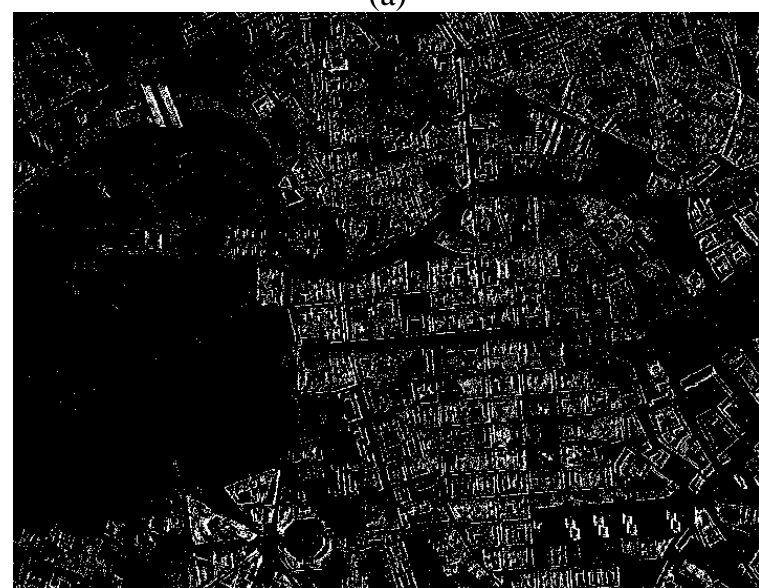

(b)

Figure 4. (a) A part of the edge image of the reference LiDAR point cloud in downtown Berlin, and (b) the edge image of the TomoSAR point cloud roughly at the same area.

\subsection{INITIAL ALIGNMENT}

The initial alignment provides an initial solution to the iterative closest point (ICP) algorithm which is known to suffer from finding possibly a local minimum. The initial alignment consists of independently finding the horizontal and the vertical shifts. The horizontal shift is found by cross-correlating the edge images of the two point clouds. In most of the cases, a unique peak can be found, due to the complex, hence pseudorandom, structures of a city. Please see Figure 5 for the 2D correlation of two edge images, where a single prominent peak is found. The vertical shift is found by cross-correlating the height histogram of the two point clouds, which is shown in Figure 6. We also set the bin spacing of the height histograms to be $2 \mathrm{~m}$ in our experiment. The accuracy of the shift estimates are of course limited by the discretization in the three directions. However, this is sufficient for the final estimation.

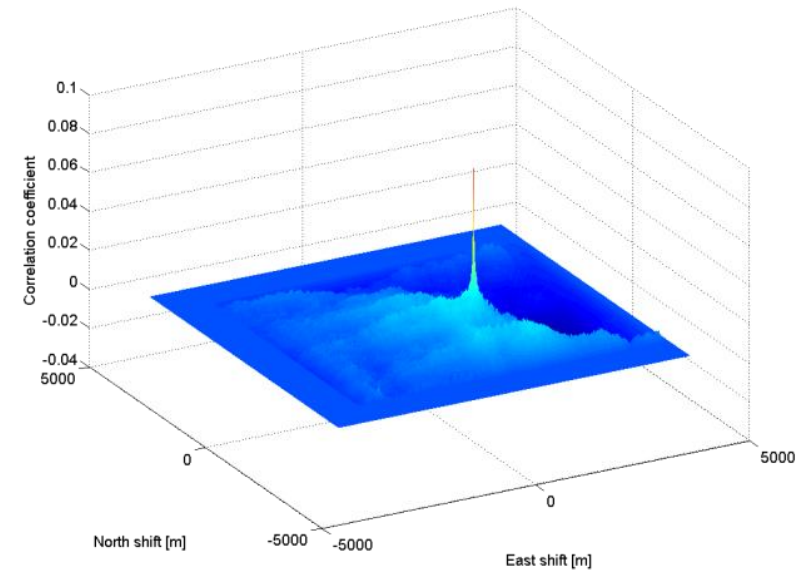

Figure 5. 2D cross-correlation of the edge images of TomoSAR and LiDAR point clouds. A single peak is found at $(828,-784) \mathrm{m}$.

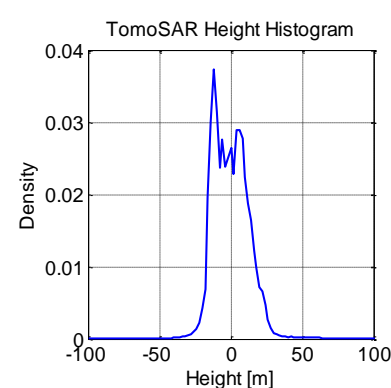

(a)

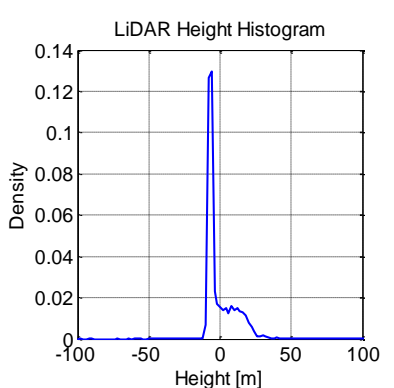

(b)

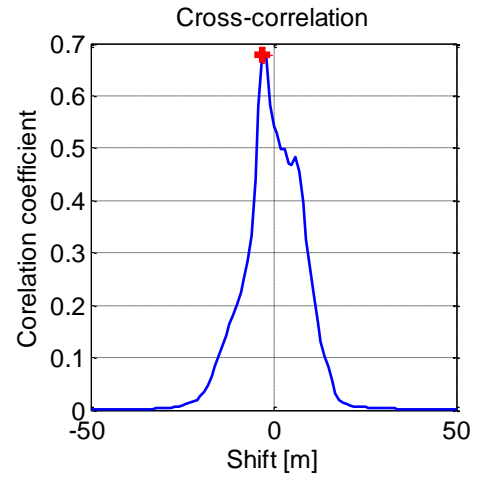

(c)

Figure 6. (a) The height histogram of TomoSAR point cloud, (b) the height histogram of LiDAR point cloud, and (c) the correlation of (a) and (b), where the red cross marks the peak position which is at $-6 \mathrm{~m}$.

\subsection{FINAL SOLUTION}

The final solution is obtained using a normal ICP algorithm based on the initial solution calculated from the previous step. The façade points in the TomoSAR point clouds are removed to prevent ICP from finding a wrong solution. The following image demonstrates the co-registered point cloud. Successful co-registration can be confirmed by seeing the correct location of the façade points in Figure 7(b). 


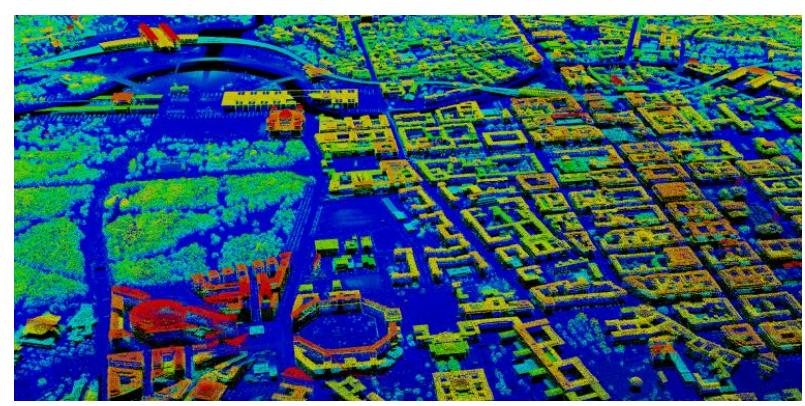

(a)

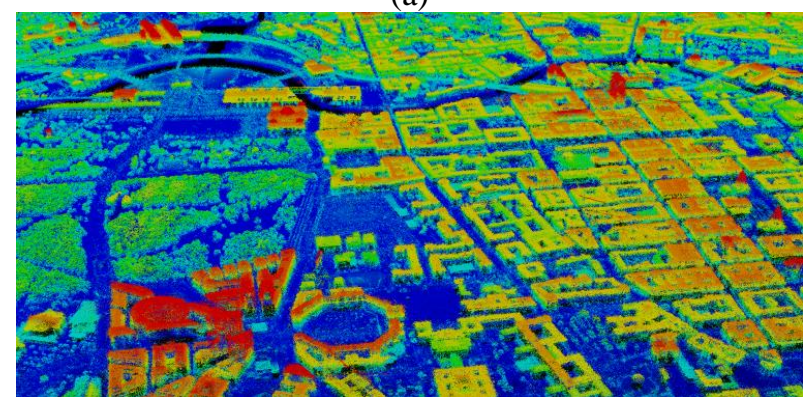

(b)

Figure 7. (a) Close up of the reference LiDAR point cloud in downtown Berlin, and (b) the co-registered point cloud combining the TomoSAR and LiDAR point cloud.

\section{COREGISTRATION OF OPTICAL IMAGE AND REFERENCE MODEL}

Currently, we rely on the fact that the optical image is already well co-registered with the reference point cloud, i.e. the camera extrinsic parameters are well known in the coordinate system of the reference point cloud. In our current experiment, the camera position is known up to an accuracy of $20 \mathrm{~cm}$ with respect to the LiDAR point cloud. However, the users are not restricted to LiDAR point cloud. One can also use a pair of stereo optical images and the reconstructed 3-D point cloud.

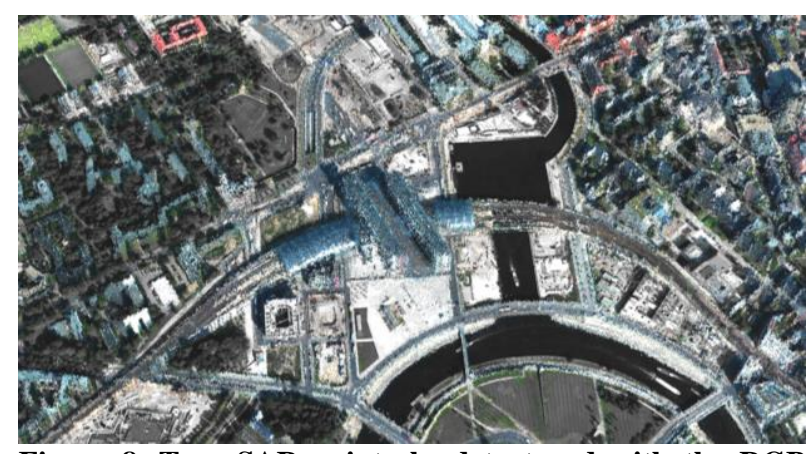

Figure 8. TomoSAR point cloud textured with the RGB color from optical image, where the dark background is the optical image not covered by the point cloud.

Figure 8 is the TomoSAR point cloud textured with RGB color from the optical image, where the subfigure (b) is the close up of the area in the dashed red rectangle in (a). Such textured point cloud enables the analysis of the SAR point cloud based on the features in optical image. Currently, we are developing algorithms for coregistering oblique optical images with 3D model, which will brings more optical information of façade points.

\section{SEMANTIC CLASSIFICATION IN OPTICAL IMAGE}

The semantic classification is done patch-wised using a dictionary-based algorithm. The entire optical image is tiled into small patches, e.g. $50 \times 50$ pixels. They are then described using a dictionary, to be specific, the occurrence of the atoms in the dictionary. Such model is known as the Bag of Words (BoW) [10]. The final patch classification is achieved using support vector machine (SVM). The detailed workflow is as follows.

\subsection{BOW MODEL}

BoW originates from text classification, where a text is modeled as the occurrence of the words in a dictionary, disregarding the grammar as well as the order. This is also recently employed in computer vision, especially in image classification. Analogous to text, the BoW descriptor $\mathbf{w}$ of an image $\mathbf{Y}$ is modeled as the occurrence of the "visual" words in a predefined dictionary $\mathbf{D}$, i.e.:

$$
\mathbf{w}=h_{\mathbf{D}}(\psi(\mathbf{Y}))
$$

where $h(\cdot)$ is the histogram operator, and $\psi(\cdot)$ is the transformation function from the image space to the feature space. Hence the visual words refer to the representative features in the image, whose ensemble constructs the dictionary.

\subsection{FEATURE EXTRACTION}

We calculate the dense local features of each patch, i.e. the feature is computed in a sliding window through the patch. This is described in Figure 9(a) where the red window traverses the patch, and computes one local feature vector at each position. The subfigure (b) is examples of some other patches extracted from the image.

Several commonly used features have been tested, which includes the most popular scale-invariant feature transform (SIFT) suggested by many literatures. However, the feature in our experiment is simply the vectorized the RGB pixel values in a $3 \times 3$ sliding window. Experiment shows its constant robustness and efficiency for large area processing.

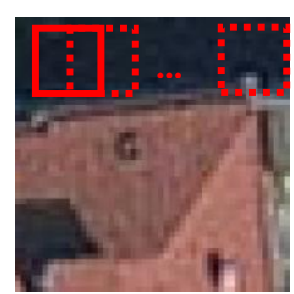

(a)
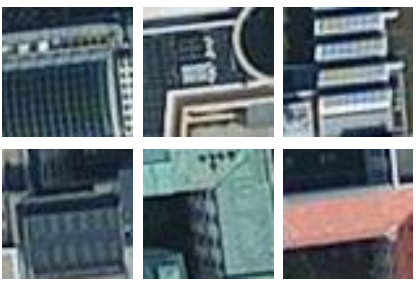

(b) 
Figure 9. (a) demonstration of dense local feature computed on an image patch, where the feature is computed in the red sliding window through the patch, and (b) some examples of other image patches.

\subsection{DICTIONARY LEARNING}

Assume the dictionary is defined as $\mathbf{D} \in \mathbb{R}^{N \times k}$, where $\mathrm{N}$ is the dimension of the word, i.e. feature vector, and $\mathrm{k}$ is the number of feature vectors, also known as atoms. The $\mathrm{k}$ feature vector should include representative features appear in the whole image, so that each patch can be well described.

Depending on the patch size, certain number of feature vectors is obtained from each patch. Collecting all of them for all the patches should already give a preliminary dictionary. However, the size of such dictionary is tremendous, knowing that an aerial optical image can be tiled into millions of patches, and each patch can give tens to hundreds of feature vectors. This renders $\mathrm{k}$ in the order of hundreds of million.

Therefore, the dimension of the preliminary dictionary should be reduced. We perform an unsupervised clustering, e.g. k-means, on the preliminary dictionary in order to quantize the feature space. The cluster center is extracted as the final dictionary. Figure 10 exemplify the quantization in a 2-D feature space. The colored crosses are the features extracted from the whole image.

\subsection{PATCH DESCRIPTOR}

The patches are described following Equation (1). Implementation-wise, this is achieved by assigning the features of a patch to their nearest neighbours in the dictionary. To this end, the patch descriptor is a vector $\mathbf{v} \in \mathbb{R}^{k}$.

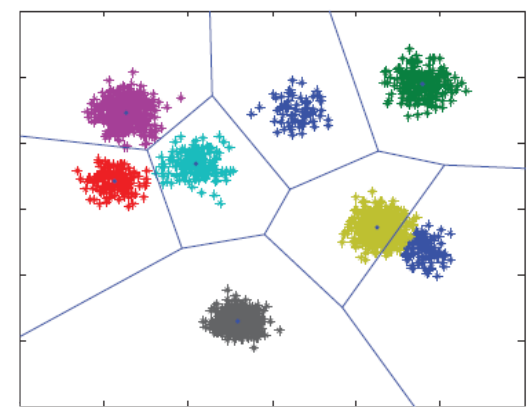

Figure 10. Demonstration of dictionary learning in two dimensional feature space. The colored crosses are the features collected from all the patches in the image. A $k$ means clustering is performed to get $k$ cluster centers, i.e. the dictionary atoms. Image modified from [11].

\subsection{CLASSIFICATION}

The classification is done using a linear SVM [12] implemented in an open source library VLFeat [13]. The SVM classifier finds a hyperplane which separates two classes of training samples with maximal margin. Giving the patch descriptor $\mathbf{v}$, its SVM classification is:

$$
f(\mathbf{v})=\operatorname{sign}\left(\mathbf{w}^{T} \mathbf{v}+b\right)
$$

where $\mathbf{w} \in \mathbb{R}^{k}$ and $b$ are the parameters of the hyperplane, and $\operatorname{sign}(\cdot)$ is the sign operator which outputs \pm 1 .

For an $m$-class $(m>2)$ problem, difference SVM should be trained for each class against the rest. The final classification of a patch $\mathbf{v}$ is assigned to the one with the largest SVM score, i.e.:

$$
f(\mathbf{v})=\max \left(\mathbf{W}^{T} \mathbf{v}+\mathbf{b}\right)
$$

where $\mathbf{W} \in \mathbb{R}^{k \times m}$ and $\mathbf{b} \in \mathbb{R}^{m}$ are the concatenated parameters of $m$ hyperplanes.

Our test image $(5000 \times 5000)$ is tiled into patches of $50 \times 50$ pixel, with 46 pixel overlap. That is to say, the classification of each patch is only assigned to the $4 \times 4$ pixel in the center. Among all the patches, 570 are manually selected as training samples. Four classes are preliminarily defined: building, roads/rail, river, and vegetation. Each of them has 240, 159, 39, and 132 training patches, respectively. The feature in our experiment is simply the vectorized RGB pixel values in a $3 \times 3$ sliding window, which results in a feature space of 27 dimension. Figure 11 shows the classification result of a region in the entire image, where the left image is the optical image, and in the right image, classified building, road, river, and vegetation are marked as red, blue, green, and blank. Despite the extremely simple feature we used, the four classes are very well distinguished.

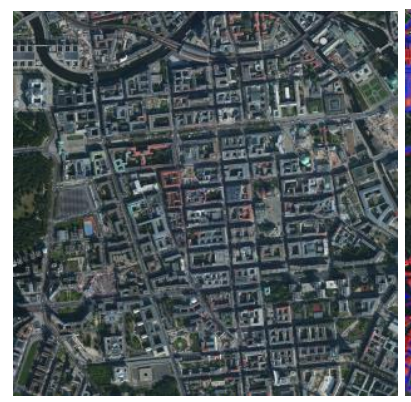

(a)

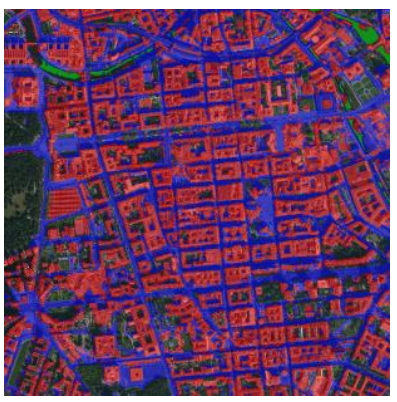

(b)
Figure 11. (a) the test optical image, and (b) the classification of building, road, river, and vegetation, where they are colored in red, blue, green, and blank.

Since we are particularly interested in building, its classification performance is evaluated by classifying half of training samples using the SVM trained with the other half of the samples. The average precision of the current algorithm is $98 \%$. The full precision and recall curve is plotted in Figure 12(a). The equivalent receiver operating characteristic curve is also shown in Figure 12(b), for the readers who are more familiar with it. The red cross marks our decision threshold which gives a detection rate of $90 \%$, and false alarm rate of $3 \%$. 


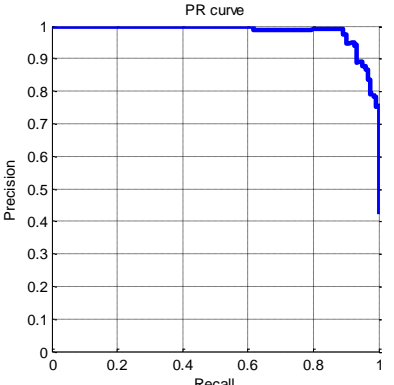

(a)

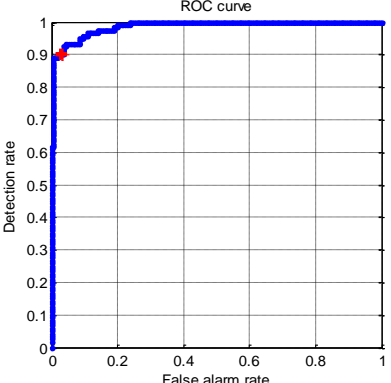

(b)
Figure 12. (a) precision and recall curve of the building classification with an average precision is $98 \%$, and (b) the ROC curve of the classification. The red cross marks our decision point which gives a detection rate of $90 \%$, and false alarm rate of $3 \%$.

\section{OBJECT-LEVEL ANALYSIS}

Based on the semantic classification, we can extend the current pixel-based monitoring and manual selection of region of interest to a systematic monitoring on an object-level. In the following, examples on bridge and railway monitoring are exemplified.

\subsection{AUTOMATIC RAILWAY MONITORING}

We applied the semantic classification scheme on an orthorectified optical image centered at the Berlin central station. We particularly classified the railway and river class for the following analysis. Figure 13 shows the classification map where the railway class and river class are labelled in green and red, respectively. The classification performance is consistent with the evaluation shown in Figure 12. Some false alarm appeared as small clusters, but they can be removed by post-processing.

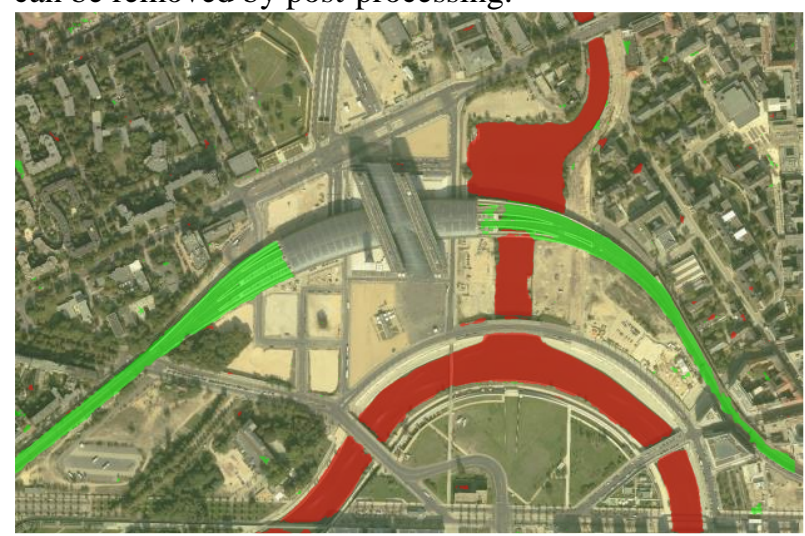

Figure 13. River (red) and railway (green) classified using the BoW method. The classification performance is consistent as the evaluation in Figure 12 shows. Some false alarm appeared as small clusters. They can be filtered out in post-processing.

Based on the classification, the corresponding points in the TomoSAR point cloud can be extracted. Assuming the railway is smooth and continuous, a smooth spline function was fitted to the $x$ and $y$ (east and north) coordinates of the railway points to connect separated segments, i.e.:

$$
\hat{\mathbf{s}}=\arg \min _{\mathbf{s}}\left\{\lambda\|\mathbf{y}-\mathbf{s}\|_{2}^{2}+(1-\lambda)\left\|\mathbf{s}^{\prime \prime}\right\|_{2}^{2}\right\}
$$

where $\mathbf{y}$ is the $y$ coordinates of the railway points, $\mathbf{s}$ is the spline function (quadratic or cubic) w.r.t. the $x$ coordinates of the railway points, and $\lambda \in[0,1]$ is the smoothing parameter. The smooth spline is centered in the railway, and the width of the railway is adaptively estimated at each position. Therefore, we are able to interpolate the discontinuity of the railway due to the presence of the Berlin central station. Figure 14(a) shows the connected railway points overlaid on a calibrated aerial image of $20 \mathrm{~cm}$ ground resolution. The color shows the amplitude of seasonal motion due to the thermal expansion of the steel. The motion parameters have been filtered by minimizing the total variation.

The seasonal motion shows a regular pattern along the railway, which is because of the expansion and contraction of individual railway section. By detecting the peaks in the derivative, the joints of railways can be detected, which are shown as the green dots in Figure 14(b). In subfigure (c), we provide the close up view of the two joints in the optical image where the railway joint shown up as dark lines are both visible.

\subsection{AUTOMATIC BRIDGE MONITORING}

By analysing the discontinuity of the river segmentation and assuming the discontinuities are caused by bridges, the bridges' positions can be detected automatically. The corresponding bridge points are extracted from the TomoSAR point cloud, and projected to the optical image.

The projected bridge points are shown in Figure 15 where the color also represents the amplitude of seasonal deformation. The upper most bridge belongs to a segment of the railway which is known to have thermal expansion. The middle bridge undergoes a $5 \mathrm{~mm}$ seasonal motion at its west end and $2 \mathrm{~mm}$ at the east end. This suggests a more rigid connection of the bridge with the foundation at its east end. The two lower bridges are stable according to the motion estimates.

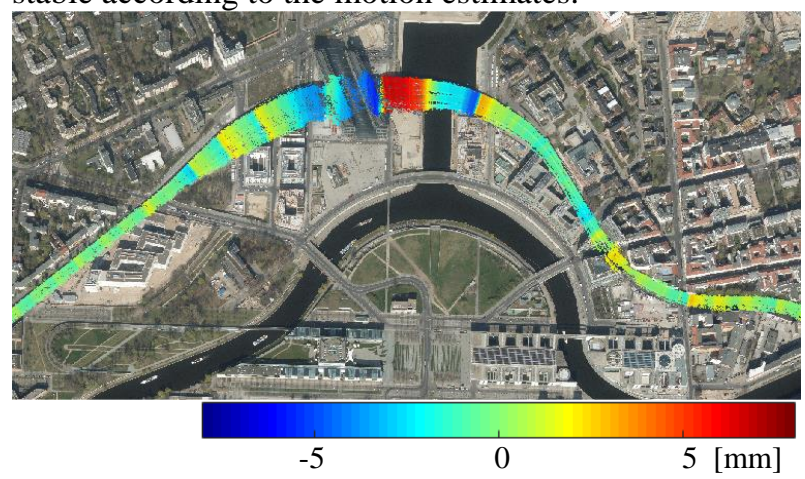

(a) 


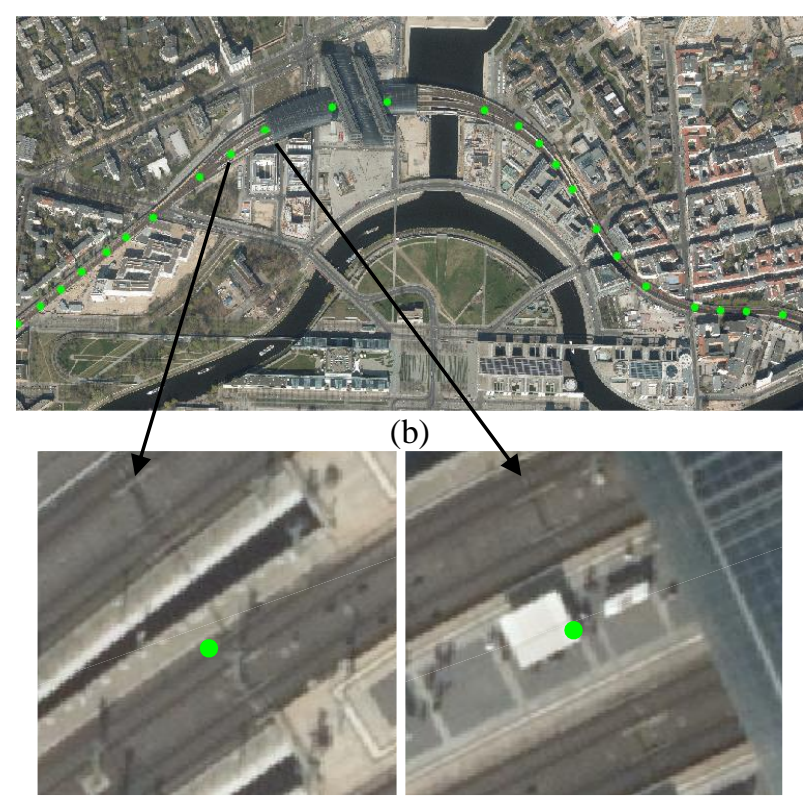

(c)

Figure 14. (a) Connected railway points extracted from the TomoSAR point cloud. The color shows the amplitude of seasonal motion due to the thermal expansion of the steel, (b) the amplitude of seasonal motion filtered by minimizing the total variation, (c) the detected railway joints marked in green, and (d)

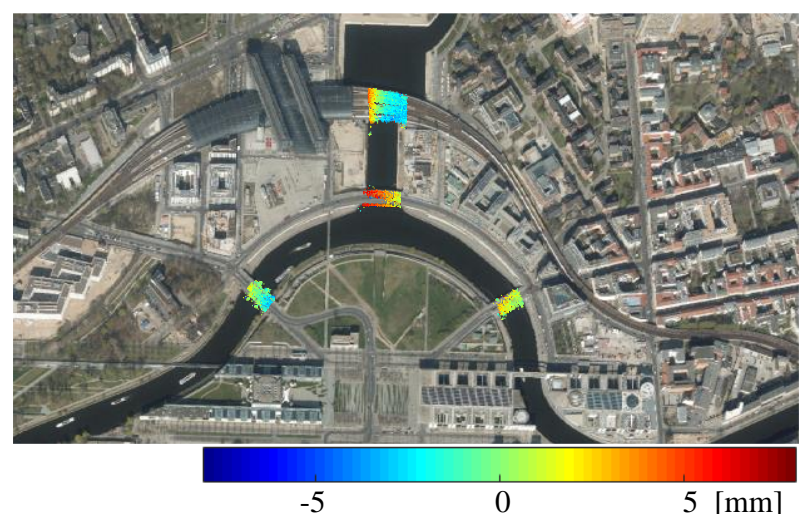

Figure 15. Overlay of the amplitude of seasonal motion of brides extracted from the TomoSAR point cloud on the optical image. The bridges are automatically detected from the classification map shown in Figure 13 using discontinuity analysis.

\section{CONCLUSION}

This paper is the first semantic analysis of high resolution InSAR point cloud in urban area. Through co-registering optical image and InSAR point cloud to a common reference 3-D model, we are able to relate the semantic meaning extracted from the optical image to the InSAR point cloud. The complementary information provided by the two data types enables an object-level InSAR deformation and 3-D analysis.

In the future, we aim at a more intelligent system by including more semantic classes, such as high-rise buildings, residential area, or even specific landmarks, and so on. To reduce the human interaction, we are also aiming at a completely unsupervised semantic classification.

\section{ACKNOWLEDGEMENT}

This work was supported by the Helmholtz Association under the framework of the Young Investigators Group "SiPEO" (VH-NG-1018, www.sipeo.bgu.tum.de), International Graduate School of Science and Engineering, Technische Universität München (Project 6.08: "4D City"), and the German Research Foundation (DFG, Förderkennzeichen BA2033/3-1)

\section{REFERENCES}

[1] R. Bamler and P. Hartl, "Synthetic aperture radar interferometry," Inverse Probl., vol. 14, no. 4, p. R1, 1998 .

[2] F. Lombardini, "Differential tomography: a new framework for SAR interferometry," IEEE Trans. Geosci. Remote Sens., vol. 43, no. 1, pp. 37-44, Jan. 2005.

[3] G. Fornaro, et al., "Four-Dimensional SAR Imaging for Height Estimation and Monitoring of Single and Double Scatterers," IEEE Trans. Geosci. Remote Sens., vol. 47, no. 1, pp. 224-237, Jan. 2009.

[4] X. X. Zhu and R. Bamler, "Very High Resolution Spaceborne SAR Tomography in Urban Environment," IEEE Trans. Geosci. Remote Sens., vol. 48, no. 12, pp. 4296-4308, 2010.

[5] X. Zhu, Very High Resolution Tomographic SAR Inversion for Urban Infrastructure Monitoring: A Sparse and Nonlinear Tour, vol. 666. Deutsche Geodätische Kommission, 2011.

[6] X. Zhu, et al., "Tomo-GENESIS: DLR's Tomographic SAR Processing System," in Urban Remote Sensing Event (JURSE), 2013 Joint, 2013, pp. 159-162.

[7] Y. Wang and X. X. Zhu, "Automatic Featurebased Geometric Fusion of Multi-view TomoSAR Point Clouds in Urban Area," IEEE J. Sel. Top. Appl. Earth Obs. Remote Sens., vol. PP, no. 99, 2014.

[8] 'Data provided by 'Land Berlin' and 'Business Location Service', supported by 'Europäischer Fonds für Regionale Entwicklung'.” .

[9] I. Sobel, "An Isotropic 3x3 Image Gradient Operator," Present. Stanf. AI Proj. 1968, 2014.

[10] G. Csurka, et al., "Visual categorization with bags of keypoints," in Workshop on statistical learning in computer vision, ECCV, 2004, vol. 1, pp. 1-2.

[11] S. Cui, "Spatial and temporal SAR image information mining," Universität Siegen, Siegen, Germany, 2014.

[12] C. Cortes and V. Vapnik, "Support-vector 
networks," Mach. Learn., vol. 20, no. 3, pp. 273297, Sep. 1995.

[13] B. F. Andrea Vedaldi, "VLFeat: an open and portable library of computer vision algorithms.," in Proceedings of the 18th International Conference on Multimedea 2010, Firenze, Italy, 2010, pp. 1469-1472. 